\title{
THE EDGE GEODETIC SELF DECOMPOSITION NUMBER OF A GRAPH
}

\author{
J. JOHN ${ }^{1, *}$ AND D. STALIN ${ }^{2}$
}

\begin{abstract}
Let $G=(V, E)$ be a simple connected graph of order $p$ and size $q$. A decomposition of a graph $G$ is a collection $\pi$ of edge-disjoint subgraphs $G_{1}, G_{2}, \ldots, G_{n}$ of $G$ such that every edge of $G$ belongs to exactly one $G_{i}(1 \leq i \leq n)$. The decomposition $\pi=\left\{G_{1}, G_{2}, \ldots, G_{n}\right\}$ of a connected graph $G$ is said to be an edge geodetic self decomposition, if $g_{e}\left(G_{i}\right)=g_{e}(G)$ for all $i(1 \leq i \leq n)$. The maximum cardinality of $\pi$ is called the edge geodetic self decomposition number of $G$ and is denoted by $\pi_{s g_{e}}(G)$, where $g_{e}(G)$ is the edge geodetic number of $G$. Some general properties satisfied by this concept are studied.
\end{abstract}

Mathematics Subject Classification. 05C12, 05C70.

Received October 13, 2019. Accepted June 26, 2020.

\section{INTRODUCTION}

By a graph $G=(V, E)$, we mean a finite undirected graph without loops or multiple edges. The order and size of $G$ are denoted by $p$ and $q$ respectively. For basic graph theoretic terminology we refer to Harary $[3,4]$. $N(v)=\{u \in V(G): u v \in E(G)\}$ is called the open neighborhood of the vertex $v$ in $G$. The degree of an vertex $v \in V(G)$ is $|N(v)|$. The maximum and minimum degree of a graph $G$ is denoted by $\Delta$ and $\delta$ respectively. A vertex of degree $p-1$ is called a universal vertex. If $e=\{u, v\}$ is an edge of a graph $G$ with $\operatorname{deg}(u)=1$ and $\operatorname{deg}(v)>1$, then we call $e$ a pendent edge, $u$ a leaf and $v$ a support vertex. For a non empty vertex subset $S \subset V(G)$ of a graph $G$, an induced sub graph of $S$ in $G$, denoted by $\langle S\rangle_{G}$, is the subgraph of $G$, with vertex set $V\left(\langle S\rangle_{G}\right)=S$ and edge set $E\left(\langle S\rangle_{G}\right)=\{u v \in E(G): u, v \in S\}$. We denote by $P_{p}, C_{p}$ and $K_{r, s}$, the path on $p$ vertices, the cycle on $p$ vertices and complete bipartite graph in which one partite set has $r$ vertices and the other partite set has $s$ vertices respectively. For any connected graph $G$, a vertex $v \in V(G)$ is called a cut vertex of $G$, if $V(G)-\{v\}$ is no longer connected. A vertex $v$ in a connected graph $G$ is said to be a semi simplicial vertex of $G$, if $\Delta(\langle N(v)\rangle)=|N(v)|-1$. A vertex $v$ is a simplicial vertex of a graph $G$, if $\langle N(v)\rangle$ is complete. Every simplicial vertex of a graph $G$ is semi simplicial vertex. A graph $G$ is said to be a semi complete graph, if every vertex of $G$ is semi simplicial. It is observed that a semi simplicial graph has no cut vertices and no end vertices. Any graph with at least two universal vertices is a semi complete graph. Also there are semi complete graphs having no universal vertex. A graph having unique universal vertex is not semi complete.

Keywords. Edge geodetic number, edge geodetic decomposition, edge geodetic self decomposition, edge geodetic self decomposition number.

1 Department of Mathematics, Government College of Engineering, Tirunelveli 627007, India.

2 Department of Mathematics, St. Alphonsa College of Arts and Science, Soosaipuram, Karungal 629157, India.

* Corresponding author: john@gcetly.ac.in 
The distance $d(u, v)$ between two vertices $u$ and $v$ in a connected graph $G$ is the length of a shortest $u-v$ path in $G$. An $u-v$ path of length $d(u, v)$ is called a $u-v$ geodesic. A vertex $x$ is said to lie on a $u-v$ geodesic $P$, if $x$ is a vertex of $P$ including the vertices $u$ and $v$. The closed interval $I_{e}[u, v]$ consist of all edges lies in $u-v$ geodesic of $G$. For $S \subseteq V, I_{e}[S]=\cup_{u, v \in S} I_{e}[u, v]$. A set $S \subseteq V$ is called an edge geodetic set of $G$, if $I_{e}[S]=E$. The edge geodetic number $g_{e}(G)$ of $G$ is the minimum order of its edge geodetic sets and any edge geodetic set of order $g_{e}(G)$ is an edge geodetic basis of $G$ or $g_{e}$-set of $G$. The edge geodetic number of a graph was introduced by M. Atici [2] and further studied in [1,6,10-14]. Recently edge geodetic concepts were studied in [9,15-17]. For any connected graph $G, 2 \leq g_{e}(G) \leq p$. For the cycle $C_{p}(p \geq 4), g_{e}\left(C_{p}\right)=2$, if $p$ is even and $g_{e}\left(C_{p}\right)=3$, if $p$ is odd. Any connected graph having exactly one universal vertex has edge geodetic number $p-1$. In this paper, we study the concepts of edge geodetic self decomposition in a connected graph $G$. Edge geodetic concepts have applications in game theory, location theory, distributed computing, information retrieval, communication networks etc.

A decomposition $\pi$ of a graph $G$ is a collection of edge-disjoint subgraphs $G_{1}, G_{2}, \ldots, G_{n}$ of $G$, such that every edge of $G$ belongs to exactly one $G_{i}(1 \leq i \leq n)$. The concept of decomposition is recently studied in $[5,7,8]$. Various types of decomposition of $G$ have been studied in the literature by imposing conditions on each subgraph $G_{i}(1 \leq i \leq n)$. Edge geodetic decomposition is very important for current research, so that the limitations with available concepts can be overcome. If each $G_{i}(1 \leq i \leq n)$ of an edge geodetic decomposition is a tree, then it speed up the computational searching. In our real life normally, design of a large scale water network with multiple source is computationally very rigorous. This is due to the size of the search space as well as the time for hydraulic simulation of the network. By considering a $g_{e}$-set as the the water source of the WDS system the edge geodetic self decomposition method to partition the larger optimization problems in to smaller ones that in turn reduces the computational overhead for optimising the design of water distribution system(WDS) $[18,19]$. Throughout this paper $G$ denotes simple connected graph with at least two vertices. The following theorems are used in the sequel.

Theorem $1.1([10])$. Each simplicial vertex, in particular every end vertex of $G$ belongs to every minimum edge geodetic set of $G$.

Theorem $1.2([10])$. For any non-trivial tree $T$, the edge geodetic number $g_{e}(T)$ equals the number of endvertices in $T$.

Theorem 1.3 ([10]). For the complete bipartite graph $G=K_{m, n}$,

$$
g_{e}\left(K_{m, n}\right)= \begin{cases}2 & \text { if } m=n=1 \\ n & \text { if } m=1, n \geq 2, \\ \min \{m, n\} & \text { if } m, n \geq 2\end{cases}
$$

\section{The EdGe Geodetic SElF DeComposition number of A GRAPH}

Definition 2.1. The decomposition $\pi=\left\{G_{1}, G_{2}, \ldots, G_{n}\right\}$ of $G$ is said to be an edge geodetic self decomposition of $G$, if $g_{e}(G)=g_{e}\left(G_{i}\right),(1 \leq i \leq n)$ for all $i$ and the maximum cardinality of $\pi$ is said to be edge geodetic self decomposition number of $G$ and is denoted by $\pi_{s g_{e}}(G)$.

Example 2.2. For the graph $G$ given in Figure $1, S=\left\{v_{1}, v_{2}, v_{3}, v_{6}\right\}$ is a minimum edge geodetic set so that $g_{e}(G)=4$. The graphs $G_{1}$ and $G_{2}$ (given in Figs. 1a and 1b) is a decomposition of $G$. Since $g_{e}\left(G_{1}\right)=g_{e}\left(G_{2}\right)=$ $4=g_{e}(G), \pi=\left\{G_{1}, G_{2}\right\}$ is an edge geodetic self decomposition of $G$. It is easily verified that there is no edge geodetic self decomposition set of cardinality more than 2 . Therefore $\pi_{s g_{e}}(G)=2$.

Definition 2.3. A graph $G$ is said to be edge geodetic self decomposable, if $\pi_{s g_{e}}(G) \geq 2$. If $G$ is not edge geodetic self decomposable graph, then $\pi_{s g_{e}}(G)=1$. 


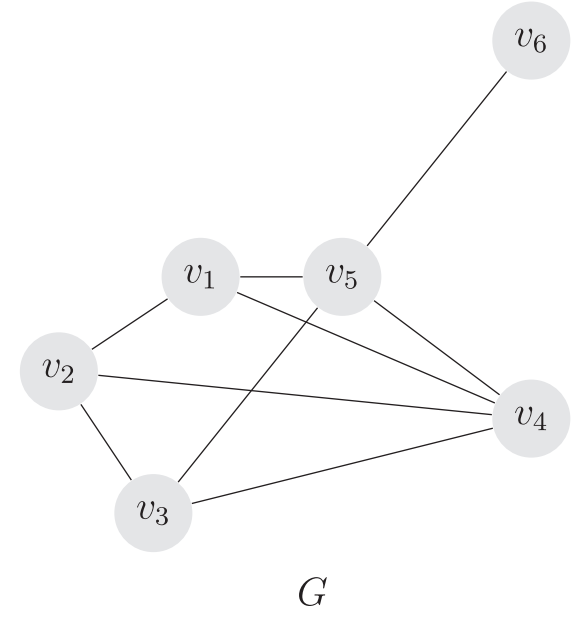

Graph having edge geodetic number 4

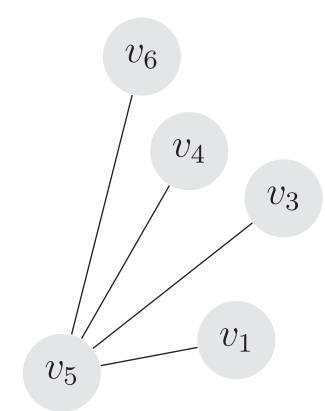

(a)

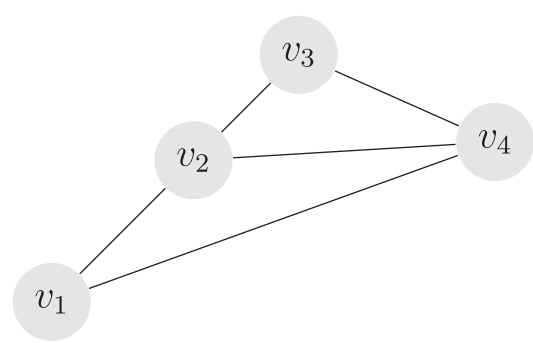

(b)

Decomposed subgraphs having edge geodetic number 4

FigURE 1.

Remark 2.4. Every graph need not be edge geodetic self decomposable. For the graph $G$ given in Figure 2, $g_{e}(G)=3$. But $G$ has no decomposition $\pi=\left\{G_{1}, G_{2}\right\}$ such that $g_{e}(G)=g_{e}\left(G_{1}\right)=g_{e}\left(G_{2}\right)=3$ and hence $\pi_{s g_{e}}(G)=1$.

Theorem 2.5. For any connected graph $G, 1 \leq \pi_{s g_{e}}(G) \leq q$.

Proof. From the Definition 2.3, $\pi_{s g_{e}}(G) \geq 1$. Let $g_{e}(G)=2$. Then $\pi(G)=\left\{G_{i}=K_{2}\right\}(1 \leq i \leq q)$. By Theorem 1.2, $g_{e}\left(G_{i}\right)=2=g_{e}(G)$. Hence $\pi$ is the unique edge geodetic self decomposition set of $G$. Therefore $1 \leq \pi_{s g_{e}}(G) \leq q$.

Remark 2.6. The bounds in Theorem 2.5 are sharp. For the graph $G$ given in Figure $2, \pi_{s g_{e}}(G)=1$ and for the path $G=P_{p}, \pi_{s g_{e}}(G)=q$. Also the bounds in Theorem 2.5 can be strict. For the graph $G$ given in Figure 1, Figures $1 \mathrm{a}$ and $1 \mathrm{~b}$ represents a maximum edge geodetic self decomposition of $G$ such that $\pi_{s g_{e}}(G)=2$. Hence $1<\pi_{s g_{e}}(G)<q$.

Theorem 2.7. Let $G$ be a connected graph with $g_{e}(G)=k(3 \leq k \leq p-2)$. Then $\pi_{s g_{e}}(G) \geq 2$, if

(i) $G$ has at least two non adjacent non pheripheral vertices $u$ and $v$ of degree $k$ such that maximum degree of each component of $\langle V-\{u, v\}\rangle \leq 2$ or 


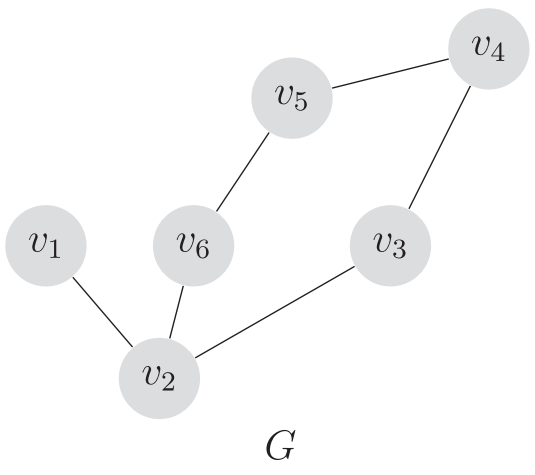

\section{Graph having edge geodetic self decomposition number 1}

\section{Figure 2.}

(ii) $\Delta(G)=k+1$ and $G$ has at least two adjacent vertices $u$ and $v$ of degree $k+1$ such that maximum degree of each component of $\langle V-\{u, v\}\rangle \leq 2$.

Proof. Case i. Suppose that $G$ has at least two non adjacent non pheripheral vertices $u$ and $v$ of degree $k$ such that maximum degree of each component of $\langle V-\{u, v\}\rangle \leq 2$.

Subcase ia. Suppose that the maximum degree of each component of $\langle V-\{u, v\}\rangle=0$. Then $g_{e}(G)=2$, which is a contradiction to $k \geq 3$. Without loss of generality let us assume that $\{u, v, w\}$ is a set of non adjacent vertices such that the components of $V-\{u, v, w\}$ are isolated vertices. Then since $\operatorname{deg}(u)=\operatorname{deg}(v)=\operatorname{deg}(w)=k, G=K_{3,3}$ and can be decomposed as exactly three star subgraphs $G_{1}=K_{1, k}, G_{2}=K_{1, k}$ and $G_{3}=K_{1, k}(k=3)$ with rooted vertices $u, v$ and $w$. Then $\pi=\left\{G_{1}, G_{2}, G_{3}\right\}$ is a decomposition of $G$. Hence by Theorem $1.2, g_{e}\left(G_{i}\right)=k,(i=1,2,3)$. Since $g_{e}(G)=k, \pi$ is an edge geodetic self decomposition of $G$.

Subcase ib. Suppose that maximum degree of each component of $\langle V-\{u, v\}\rangle$ is either 1 or 2. Assume that $\{u, v\}$ is a cut set of $G$. Let $G_{l_{1}}, G_{l_{2}}, \ldots, G_{l_{n}}$ be the components of $\langle V-\{u, v\}\rangle$. Since $1 \leq[\langle\Delta(V-$ $\{u, v\})\rangle] \leq 2$, at least one component $G_{l_{i}}(1 \leq i \leq n)$ is a path. Let $u_{l_{i}}, u_{l_{i+1}}, \ldots, u_{l_{j}}=G_{l_{i}}$ be the path. Then either $u, v$ or both adjacent with $u_{l_{i}}$ and $u_{l_{j}}$. Also $G_{1}=\left[\left\langle u \cup N(u) \cup\left\{u_{l_{i}}, u_{l_{i+1}}, \ldots, u_{l_{j}}\right\}\right\rangle\right]$ and $G_{2}=[\langle v \cup N(v)\rangle]$ or $G_{1}=\left[\left\langle v \cup N(v) \cup\left\{u_{l_{i}}, u_{l_{i+1}}, \ldots, u_{l_{j}}\right\}\right\rangle\right]$ and $G_{2}=[\langle u \cup N(u)\rangle]$ are trees having $k$ end vertices. Now $\pi=\left\{G_{1}, G_{2}\right\}$ is a decomposition of $G$ and by Theorem 1.2, $g_{e}\left(G_{1}\right)=g_{e}\left(G_{2}\right)=g_{e}(G)=k$ so that $\pi$ is an edge geodetic self decomposition of $G$ and hence $\pi_{s g_{e}}(G) \geq 2$.

Suppose that $\{u, v\}$ is not a cut set of $G$. Since $1 \leq \Delta(\langle V-\{u, v\}\rangle) \leq 2,\langle V-\{u, v\}\rangle$ is path. Let $u_{i}, u_{i+1}, \ldots, u_{j}$ be the path. Then $\pi=\left\{G_{1}, G_{2}\right\}$ is a decomposition of $G$, where $G_{1}=\left[\left\langle u \cup N(u) \cup\left\{u_{1} u_{2}\right\}\right\rangle\right]$ and $G_{2}=\left[\left\langle v \cup N(v) \cup\left\{u_{2}, u_{3}, \ldots, u_{j}\right\}\right\rangle\right]$. Moreover $G_{1}$ and $G_{2}$ are trees and has $k$ end vertices. Hence by Theorem 1.2, $g_{e}\left(G_{1}\right)=g_{e}\left(G_{2}\right)=g_{e}(G)=k$ so that $\pi$ is an edge geodetic self decomposition of $G$. Thus $\pi_{s g_{e}}(G) \geq 2$.

Case ii. Without loss of generality assume that $u$ and $v$ are the two adjacent vertices of $G$ having degree $k+1$. Let $G_{1}=K_{1, k}$ be a star graph with rooted vertex $v$. Since $\triangle(G)=k+1, G_{2}=G-\{v\}$ is a graph having exactly one vertex of degree $k$. Then as in the Case i, $\pi(G)=\left\{G_{1}, G_{2}\right\}$ is an edge geodetic self decomposition of $G$ so that $\pi_{s g_{e}}(G) \geq 2$.

Remark 2.8. The converse of the Theorem 2.7 need not be true. For the graph $G$ given in Figure 3 , $S=\left\{v_{1}, v_{2}, v_{5}\right\}$ is the minimum edge geodetic set of $G$ so that $g_{e}(G)=k=3$. However $G$ can be decomposed as $G_{1}$ and $G_{2}$ given in Figures $3 \mathrm{a}$ and $3 \mathrm{~b}$, each has edge geodetic number 3 and $G$ has only one vertex $v_{3}$ of degree 3 and adjacent vertex $v_{8}$ has degree $5 \neq k+1$. 


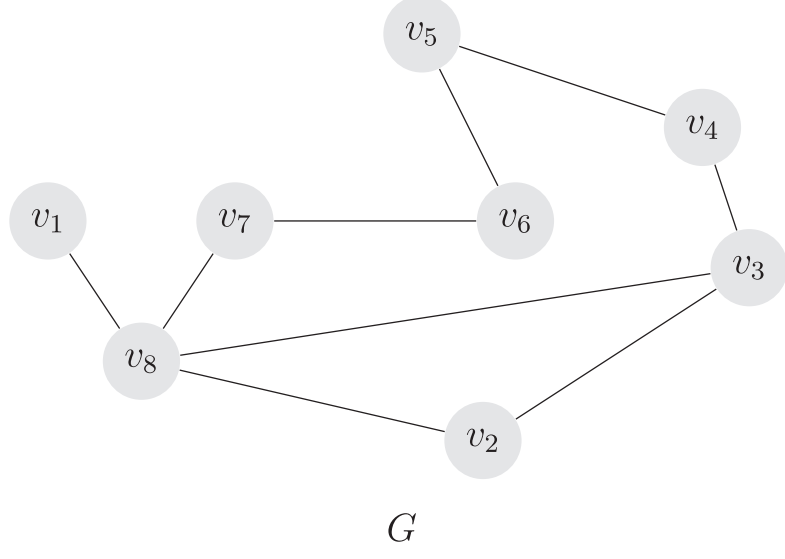

Edge geodetic self decomposable graph having edge geodetic number 3 and unique vertex of degree 3

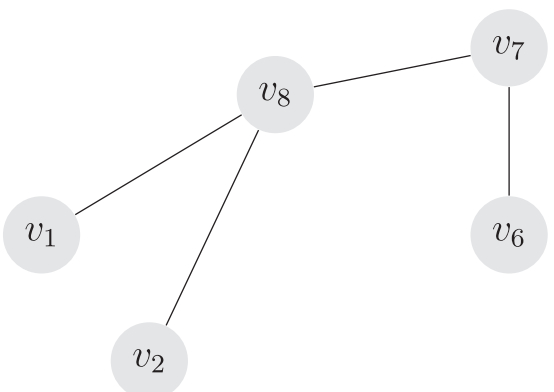

$G$

(a)

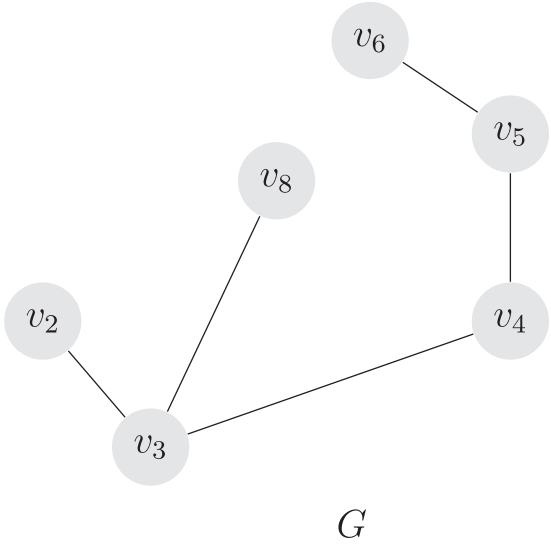

(b)

Decomposed subgraphs having edge geodetic number 3

FiguRE 3.

Theorem 2.9. Let $G$ be an edge geodetic self decomposable graph with $g_{e}(G)=k(3 \leq k \leq p-2)$ and $G$ has $r$ non adjacent vertices of degree $k$. Then $\pi_{s g_{e}}(G) \geq r$.

Proof. Let $S=\left\{u_{1}, u_{2}, \ldots, u_{r}\right\}$ be the set of $r$ non adjacent vertices of degree $k$.

Case i. Suppose that each component of $\langle V-S\rangle$ is $K_{1}$. Then it is easily verified that $\pi=\left\{G_{1}, G_{2}, \ldots, G_{r}\right\}$ is an edge geodetic self decomposition of $G$, where $G_{i}=K_{1, k}(1 \leq i \leq r)$ and so $\pi_{s g_{e}}(G)=r$.

Case ii. Suppose that $\Delta(\langle V-S\rangle) \geq 2$.

Subcase iia. Suppose that the components of $\langle V-S\rangle$ are paths and $S$ dominates $G$. Then as the same arguments given in Theorem 2.7, $\pi_{s g_{e}}(G)=r$.

Subcase iib. Suppose that at least one component of $\langle V-S\rangle$ is not a path. Since $G$ is an edge geodetic self decomposable, the non path component has edge geodetic number $k$ (otherwise $G$ cannot be edge geodetic self decomposable). Hence $\pi_{s g_{e}}(G) \geq r$. 


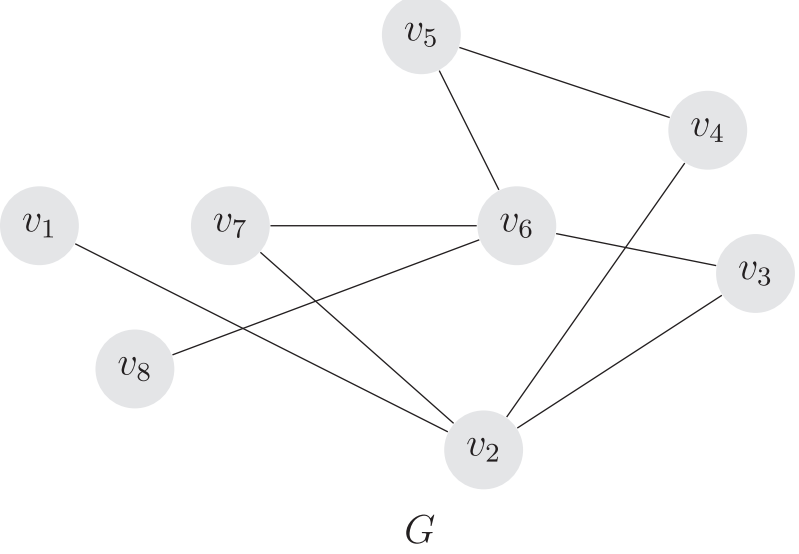

Graph having edge geodetic number 3, edge geodetic self decomposition number 2 and has no vertices of degree 3

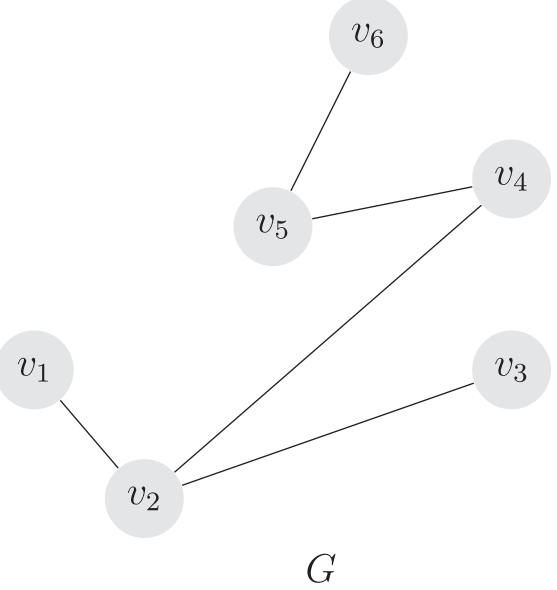

(a)

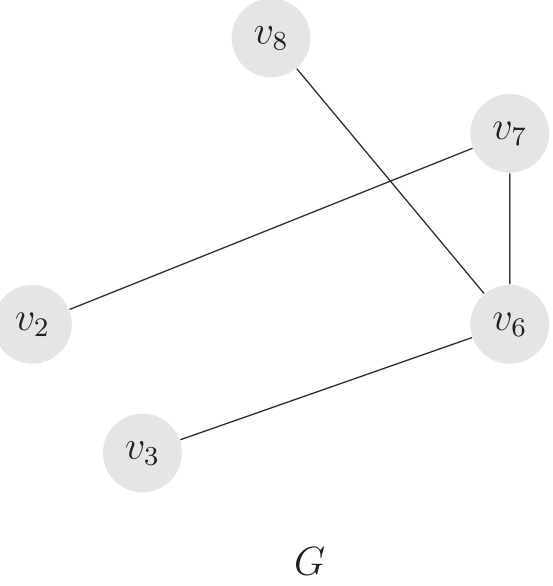

(b)

Decomposed subgraphs having edge geodetic number 3

Figure 4.

Remark 2.10. The converse of the Theorem 2.9 need not be true. For the graph $G$ given in Figure 4 , $S=\left\{v_{1}, v_{5}, v_{8}\right\}$ is a minimum edge geodetic set of $G$ so that $g_{e}(G)=k=3$. However $G$ can be decomposed as $G_{1}$ and $G_{2}$ given in Figures $4 \mathrm{a}$ and $4 \mathrm{~b}$, each has edge geodetic number 3 and $G$ has no vertex of degree 3 .

Theorem 2.11. Let $G$ be a k-regular graph, where $g_{e}(G)=k(3 \leq k \leq p-2)$. Then $\pi_{s g_{e}}(G) \geq \beta(G)$.

Proof. This follows from Theorem 2.9 .

Theorem 2.12. Let $G$ be a $k$-regular graph where $g_{e}(G)=k(3 \leq k \leq p-2)$ and $S$ be an independent set of $G$. Then $\pi_{\text {sge }}(G)=\beta(G)$, if and only if $S$ is a dominating set of $G$ as well as maximum degree of each component of $\langle V-S\rangle \leq 2$.

Proof. Let $S=\left\{u_{1}, u_{2}, \ldots, u_{r}\right\}$ be an independent set of $G$. Suppose that $\pi_{s g_{e}}(G)=\beta(G)$. We have to prove $S$ is a dominating set of $G$ and maximum degree of each component of $\langle V-S\rangle \leq 2$. 
Case i. Suppose that $S$ is not a dominating set of $G$. Then, there exists at least one vertex $v \in V$ such that $v \notin N(S)$. Without loss of generality let us assume that $v$ is adjacent to $w$, where $w \in N\left(u_{i}\right)(1 \leq i \leq r)$. As the same argument in the Theorem 2.7, let $u_{i}$ be the rooted vertex of $G_{i} \in \pi$. Hence, either $g_{e}\left(G_{i}\right) \neq k$ or $G$ is not edge geodetic self decomposable so that $\pi_{s g_{e}}(G) \neq \beta(G)$, which is a contradiction.

Case ii. Suppose that maximum degree of each component of $\langle V-S\rangle \leq 2$. Then each component of $\langle V-S\rangle$ is neither $\bar{K}_{|v-s|}$ nor a path. If $S$ is a cut set of $G$, then at least one component of $\langle V-S\rangle$ has degree at least three. Also, if $S$ is not a cut set of $G$, then $\Delta[\langle V-S\rangle] \geq 3$. Since $G$ is edge geodetic self decomposable, $g_{e}[\langle V-S\rangle]=k$ or each component of $V-S$ has edge geodetic number $k$ (otherwise $G$ cannot be edge geodetic self decomposable) so that $\pi_{s g_{e}}(G) \neq \beta(G)$, which is a contradiction.

Conversely suppose that $S$ is a dominating set of $G$ and maximum degree of each component of $\langle V-S\rangle \leq 2$. Then by Theorem $2.9, \pi_{s g_{e}}(G) \geq \beta(G)$. Suppose that $\pi_{s g_{e}}(G)>\beta(G)$. Let $\beta=\left\{u_{1}, u_{2}, \ldots, u_{r}\right\}$ be a maximum independent set of $G$. Then the maximum degree of $\langle V-\beta\rangle \geq 3$, which is a contradiction. Therefore $\pi_{s g_{e}}(G)=\beta(G)$.

Remark 2.13. The Theorem 2.12 need not be true, if $G$ is a non regular graph. For the graph $G=K_{3,4}$, $\pi_{s g_{e}}(G)=\beta(G)=4$. However $G$ is not a regular graph.

Theorem 2.14. Let $G$ be an edge geodetic self decomposable graph and $g_{e}(G)=k(k \neq 2)$. Then $\pi_{s g_{e}}(G) \leq\left\lceil\frac{q}{k}\right\rceil$. Moreover $\pi_{s g_{e}}(G)=\frac{q}{k}$, if and only if $G_{i}=K_{1, k},(1 \leq i \leq n),(k \neq 3)$ and for $k=3, G_{i}=K_{1,3}$ or $K_{3},(1 \leq i \leq n)$.

Proof. Let $G$ be an edge geodetic self decomposable graph and $g_{e}(G)=k$ and $G_{1}, G_{2}, \ldots, G_{n} \in \pi$. Then $g_{e}\left(G_{i}\right)=k,(1 \leq i \leq n)$ so that $\left|E\left(G_{i}\right)\right| \geq k$. Therefore $\pi_{s g_{e}}(G) \leq\left\lceil\frac{q}{k}\right\rceil$. Suppose that $\pi_{s g_{e}}(G)=\frac{q}{k}$ and $G_{i} \neq K_{1, k}$. Then $E\left(G_{i}\right)>k$ and so $\pi_{s g_{e}}(G)<\frac{q}{k}$, which is a contradiction. Moreover if $G_{i}=K_{1,3},(1 \leq i \leq n-1)$ or $G_{i}=K_{3}, E\left(G_{i}\right)=k(1 \leq i \leq n)\left(\right.$ or $\left.G_{i}=K_{3}, G_{j}=K_{1,3}(1 \leq i, j \leq n)\right)$. Then it is clear that $\pi_{s g_{e}}(G)=\frac{q}{k}$. Converse is clear.

Corollary 2.15. For the wheel graph $G=W_{1, p-1}(p \geq 3), \pi_{s g_{e}}(G)=1$.

Proof. For the wheel graph $G=W_{1, p-1}(p \geq 4), q=2(p-1)$ and hence $g_{e}(G)=p-1$. Also by Theorem 2.14, $\pi=\left\{G_{1}, G_{2}\right\}$ and $G_{1}=G_{2}=K_{1, p-1}$. Since $G$ contains only one universal vertex this decomposition is not possible. Hence it follows that $\pi_{s g_{e}}(G)=1$.

Theorem 2.16. For a connected graph $G, \pi_{s g_{e}}(G)=q$, if and only if $g_{e}(G)=2$.

Proof. Let $\pi_{s g_{e}}(G)=q$. Then it is clear that $\pi=\left\{G_{i}=K_{2}\right\},(1 \leq i \leq q)$. Hence by Theorem $1.2, g_{e}(G)=2$. Conversely suppose that $g_{e}(G)=2$. We have to prove $\pi_{s g_{e}}(G)=q$. It is enough to prove that $\pi=\left\{G_{i}=K_{2}\right\}$, $(1 \leq i \leq q)$. Suppose that $G_{i} \neq K_{2}$ for some $i(1 \leq i \leq q)$. Then $\left|E\left(G_{i}\right)\right| \geq 2$ for some $i,(1 \leq i \leq q)$. Hence, it follows that $\pi_{s g_{e}}(G)<q$, which is a contradiction. Therefore $\pi_{s g_{e}}(G)=q$.

Corollary 2.17. For an even cycle $G=C_{2 n}(n \geq 2), \pi_{s g_{e}}(G)=q$.

Proof. This follows from Theorem 2.16.

Observation 2.18. Let $G \neq C_{p}$ be a unicyclic graph with edge geodetic number $g_{e}(G)=k(3 \leq k \leq p-3)$. Then $G$ has at least $k-2$ end vertices.

Theorem 2.19. Let $G \neq C_{p}$ be a unicyclic graph. Then $\pi_{s g_{e}}(G) \geq 2$, if and only if $G$ is the anyone of the graph given in the family $\Im$.

Proof. Let $G \neq C_{p}$ be a unicyclic graph and $C$ be the cycle in $G$ such that $|C|=C(G)$. 
Edge geodetic self decomposable unicyclic graphs having edge geodetic number 4

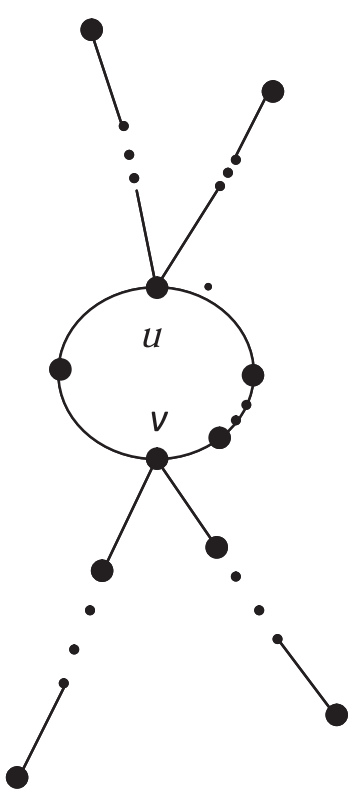

$G$

(a) $u$ and $v$ are peripheral vertices

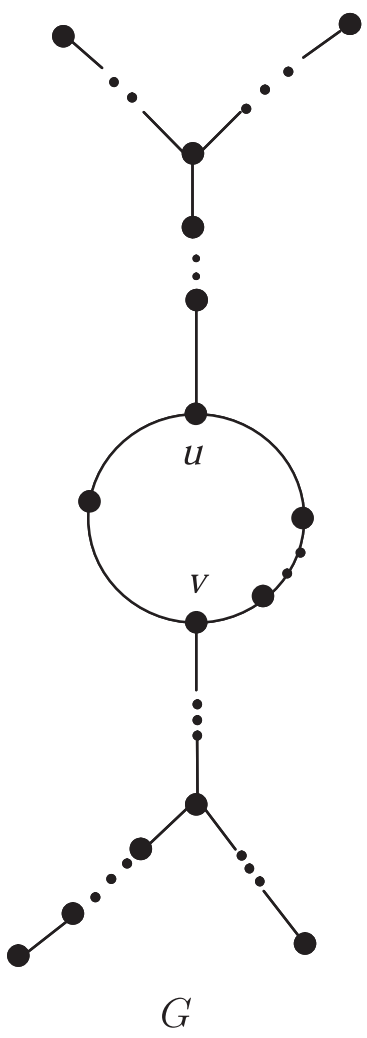

(b)

FiguRE 5. The family of graph $\Im$.

Case i. Suppose that $g_{e}(G)=k,(5 \leq k \leq p-1)$. Then by Observation 2.18, $G$ has at least $k-2$ end vertices. If $\pi_{s g_{e}}(G)=r(r \geq 2)$, then each decomposition indices graph $G_{i}(1 \leq i \leq r)$ has at least $k-2$ end vertices of $G$. Hence $G$ has at least $r(k-2)$ end vertices and so $g_{e}(G) \geq r(k-2)$, which is a contradiction.

Case ii. Suppose that $g_{e}(G)=4$. Then by Theorem 1.1, $G$ has at most four end vertices. Let $S=\left\{v_{1}, v_{2}, v_{3}, v_{4}\right\}$ be the set of all end vertices.

Subcase iia. Suppose that $C(G)$ is odd and any two non adjacent vertices has degree four. Then there exists at least one edge $e$ of $G$ such that $e \notin I_{e}[S]$. Hence, it follows that $S$ is not an edge geodetic set of $G$ so that $g_{e}(G)>4$, which is a contradiction.

Subcase iib. Suppose that $C(G)$ is even and $\Delta(G)=4$. Let $u$ and $v$ be the pair of peripheral vertices of $C$. If at least one of the peripheral vertex of $G$ has degree 2, then there exists at least one edge $e$ of $G$ such that $e \notin I_{e}[S]$. Hence, it follows that $S$ is not an edge geodetic set of $G$ so that $g_{e}(G)>4$, which is a contradiction.

Subcase iic. Suppose that $C(G)$ is even and $G$ has exactly three end vertices. Then it is clear that $\pi_{s g_{e}}(G)=1$. Thus, if $g_{e}(G)=4$, then only possible unicyclic edge geodetic self decomposable graph $G$ are given in Figures $5 \mathrm{a}$ and $5 \mathrm{~b}$ in the family $\Im$. The graphs $G$ of $\Im$ given in Figures $5 \mathrm{a}$ and $5 \mathrm{~b}$ can be decomposed as $G_{1}$ and $G_{2}$ such that each graph is a tree with four end vertices. Hence by Theorem $1.2, g_{e}\left(G_{1}\right)=$ $g_{e}\left(G_{2}\right)=g_{e}(G)=4$ so that $\pi_{s g_{e}}(G) \geq 2$. 
Edge geodetic self decomposable unicyclic graphs having edge geodetic number 3

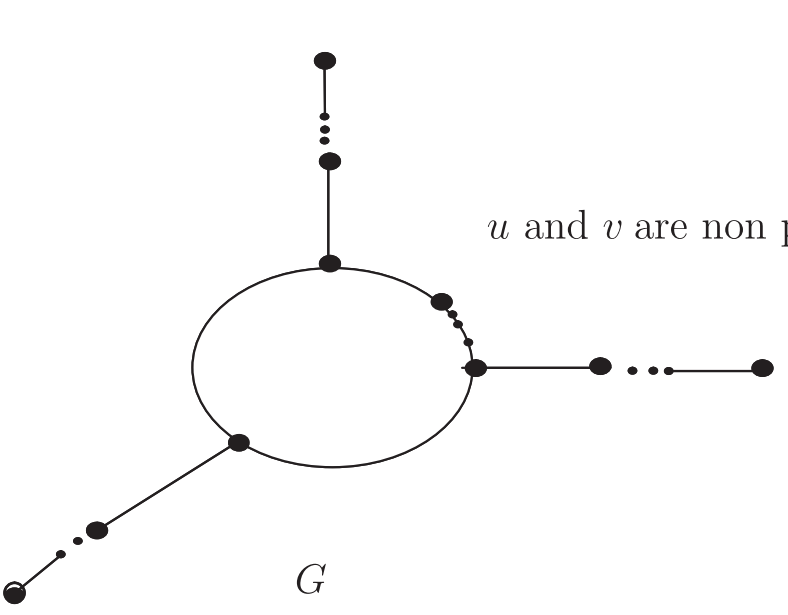

(c)

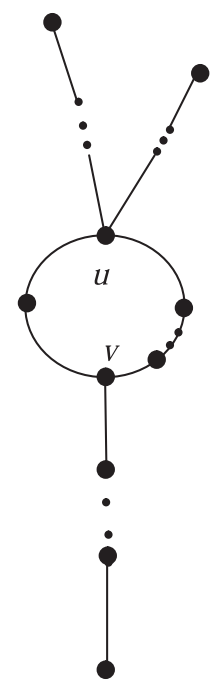

G

(e)

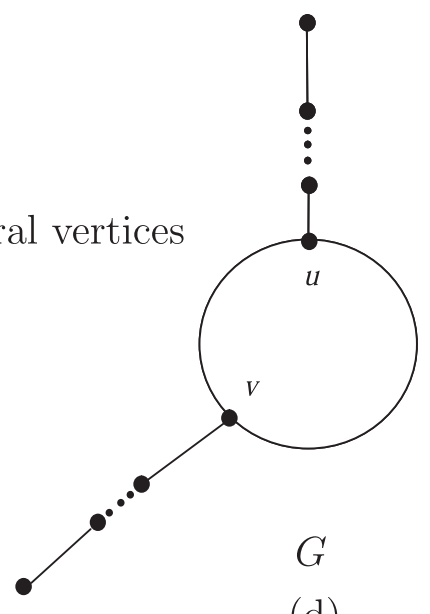

(d)

$u$ and $v$ are peripheral vertices

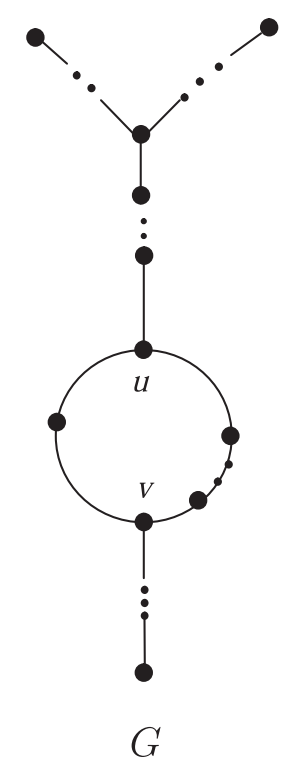

(f)

Figure 5. (Continued.) 
Edge geodetic self decomposable unicyclic graphs having edge geodetic number 2

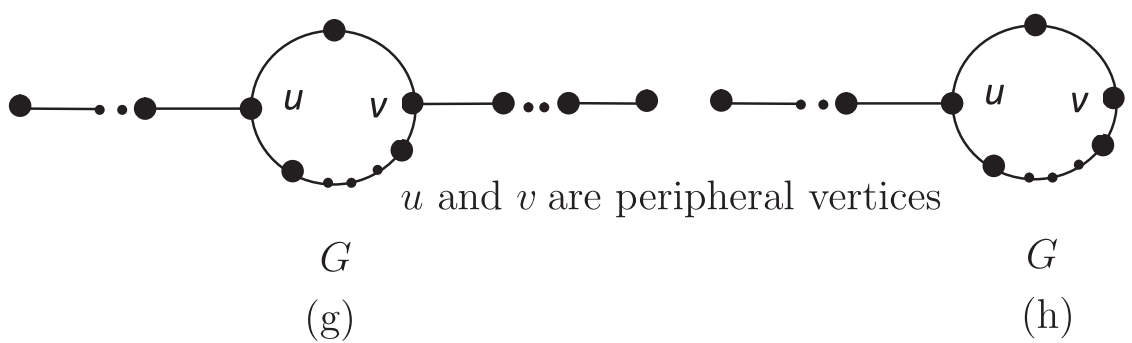

Figure 5. (Continued.)

Case iii. $g_{e}(G)=3$. Then by Theorem 1.1, $G$ has at most three end vertices. Let $S=\left\{v_{1}, v_{2}, v_{3}\right\}$ be the set of all end vertices of $G$.

Subcase iiia. Suppose $G$ has exactly three vertices $u, v$ and $w$ of degree 3 such that $d(u, w)>d(u, v)+$ $d(v, w)$. Then it is clear that $G$ has at least one edge $e$ of $G$ such that $e \notin I_{e}[S]$. Hence, it follows that $S$ is not an edge geodetic set of $G$ so that $g_{e}(G)>3$, which is a contradiction.

Subcase iiib. Suppose $C$ is an even cycle with at least one non peripheral vertex has degree three. Then $G$ has an edge $e$ such that $e \notin I_{e}[S]$. Hence, it follows that $S$ is not an edge geodetic set of $G$ so that $g_{e}(G)>3$, which is a contradiction. Moreover, if $G$ has exactly one vertex of degree 3 . Hence it follows that $\pi_{s g_{e}}(G)=1$.

Subcase iiic. Suppose $C(G)$ is even and exactly one non peripheral vertex has degree four. Then there exists at least one edge $e$ of $G$ such that $e \notin I_{e}[S]$. Hence, it follows that $S$ is not an edge geodetic set of $G$ so that $g_{e}(G)>3$, which is a contradiction.

Subcase iiid. Suppose $G$ has exactly two end vertices $u$ and $v$. If any two peripheral vertices of $G$ has degree three, then $g_{e}(G)=2$, which is a contradiction. Thus, if $g_{e}(G)=3$, then only possible unicyclic edge geodetic self decomposable graphs $G$ are given in Figures $5 \mathrm{c}-5 \mathrm{f}$ in the family $\Im$. The graphs $G$ of $\Im$ given in Figures $5 \mathrm{c}-5 \mathrm{f}$ can be decomposed as $G_{1}, G_{2}$ or $G_{3}$ such that each graph is a tree with three end vertices. Hence by Theorem $1.2, g_{e}\left(G_{i}\right)=g_{e}(G)=3(1 \leq i \leq 3)$ so that $\pi_{s g_{e}}(G) \geq 2$.

Case iv. Suppose that $g_{e}(G)=2$. Then by Theorem 1.1, G has at most two end vertices and $C(G)$ must be even. Hence at most two peripheral vertices of $G$ can have degree three. Thus, if $g_{e}(G)=2$, then only possible unicyclic edge geodetic self decomposable graphs $G$ are given in Figures $5 \mathrm{~g}$ and $5 \mathrm{~h}$ in the family $\Im$. Then by Theorem $2.16, \pi_{s g_{e}}(G) \geq 2$.

Corollary 2.20. Let $G$ be a unicyclic graph with $g_{e}(G) \neq 2$. Then $\pi_{s g_{e}}(G) \leq 3$.

Proof. This follows from Theorem 2.19.

The following theorem was proved in [5].

Theorem A. For a connected graph $G, g_{e}(G)=p$, if and only if $G$ is semi complete.

Theorem 2.21. Let $G$ be a connected graph with $g_{e}(G)=p$. Then $\pi_{s g_{e}}(G)=1$.

Proof. Suppose that $G$ is an edge geodetic self decomposable graph with $g_{e}(G)=p$. Then by Theorem A, $G$ is semi complete. Let $\pi=\left\{G_{1}, G_{2}, \ldots, G_{n}\right\}$ be a edge geodetic self decomposition of $G$. Then $g_{e}(G)=g_{e}\left(G_{i}\right)(1 \leq$ $i \leq n)$. Since $g_{e}(G)=p, g_{e}\left(G_{i}\right)=p(1 \leq i \leq n)$. Then each $G_{i}(1 \leq i \leq n)$ is semi complete. Therefore each $G_{i}(1 \leq i \leq n)$ has either at least two universal vertices or $G$ has no universal vertices. 
Case i. Suppose that $G_{1}$ (say) has at least two universal vertices. Hence any one of $G_{i}(2 \leq i \leq n)$ has order at most $p-2$. Then $g_{e}\left(G_{i}\right) \leq p-2(2 \leq i \leq n)$, which is a contradiction. Therefore $\pi_{s g_{e}}(G)=1$.

Case ii. Suppose that $G_{1}$ (say) has no universal vertices. Then at least two vertices of $G$ has degree $p-2$. Hence anyone of $G_{i}(2 \leq i \leq n)$ has at least two pendent vertices (otherwise $V\left(G_{i}\right) \leq p-1(2 \leq i \leq n)$ ). Hence $G_{i}(2 \leq i \leq n)$ is not semi complete, which is a contradiction. Therefore $\pi_{s g_{e}}(G)=1$.

Corollary 2.22. Let $G$ be a connected graph having more than one universal vertex. Then $\pi_{s g_{e}}(G)=1$.

Proof. This follows from Theorem 2.21.

Corollary 2.23. For the complete graph $G=K_{p}(p \geq 3), \pi_{s g_{e}}(G)=1$.

Proof. This follows from Corollary 2.22 .

Theorem 2.24. Let $G$ be a connected graph with edge geodetic number $p-1$. Then $\pi_{s g_{e}}(G)=2$, if $G=K_{1}+H$, where $H$ is a semi complete graph of order $p-1$.

Proof. Let $G=K_{1}+H$, where $H$ is a semi complete graph of order $p-1$. Then $G$ can be decomposed as $G_{1}=K_{1, p}$ and $G_{2}=H$ and hence by Theorems 1.2 and $\mathrm{A}, \pi_{s g_{e}}(G) \geq 2$. Moreover by Theorem 2.21, $\pi_{s g_{e}}(G)=1$ so that $\pi_{s g_{e}}(G)=2$.

Theorem 2.25. For the complete bipartite graph $G=K_{m, n}(1 \leq m \leq n)$,

$$
\pi_{s g_{e}}\left(K_{m, n}\right)= \begin{cases}1 & \text { if } m=1 \\ 2 n & \text { if } m=2, n \geq 3 \\ n & \text { if } m, n \geq 3\end{cases}
$$

Proof. Case i. Suppose that $m=1$. Then $G=K_{1, n}$. Hence by Theorem $2.14, \pi_{s g_{e}}(G)=1$.

Case ii. Suppose that $m=2$. Then by Theorems 1.3 and 2.16, $\pi_{s g_{e}}(G)=2 n$.

Case iii. Suppose that $3 \leq m \leq n$. Let $U=\left\{u_{1}, u_{2}, \ldots, u_{m}\right\}$ and $W=\left\{w_{1}, w_{2}, \ldots, w_{n}\right\}(m \leq n)$ be a bi-partition of $G$. Then by Theorem 1.3, $g_{e}(G)=m$. Since $\operatorname{deg}\left(w_{i}\right)=m(1 \leq i \leq n) G$ can be decomposes to $n$ subgraphs $G_{i}=K_{1, m}(1 \leq i \leq n)$ with rooted vertex $w_{i}(1 \leq i \leq n)$ so that $\pi=\left\{G_{1}, G_{2}, \ldots, G_{n}\right\}$ is a decomposition of $G$. Then by Theorem 1.2, $g_{e}\left(G_{i}\right)=m=g_{e}(G)$ so that $\pi$ is an edge geodetic self decomposition of $G$. Since each $G_{i}$ is a star, $\pi$ is an edge geodetic self decomposition of $G$. Therefore $\pi_{s g_{e}}(G)=n$.

Lemma 2.26. Let $G=K_{r_{1}, r_{2}, r_{3}, \ldots, r_{n}}$ be a complete $n$-partite graph with $r_{1} \leq r_{2} \leq r_{3} \leq, \ldots, \leq r_{n}$. Then

$$
g_{e}(G)=\sum_{i=1}^{n-1} r_{i}
$$

Proof. Let us prove the theorem by induction on $i$. Suppose $i=2$. Then by Theorem 1.3, the result is true. Let $V_{1}=\left\{v_{11}, v_{12}, \ldots, v_{1 r_{1}}\right\}, V_{2}=\left\{v_{21}, v_{22}, \ldots, v_{2 r_{2}}\right\}, \ldots, V_{k}=\left\{v_{k 1}, v_{k 2}, \ldots, v_{k r_{k}}\right\}$ be the partition of $V$ of a complete $k$ - partite graph $G^{\prime}$ such that $r_{1} \leq r_{2} \leq r_{3} \leq \ldots \leq r_{k}$. Then by induction hypothesis $g_{e}(G)=\sum_{i=1}^{k-1} r_{i}$. Let $V_{k+1}=\left\{v_{(k+1) 1}, v_{(k+1) 2}, \ldots, v_{(k+1) r_{(k+1)}}\right\}$. Then $G=G^{\prime}+V_{k+1}$ is a complete $k+1-$ partite graph and

$$
d\left(v_{i j}, v_{r s}\right)= \begin{cases}1 & \text { if } i \neq r \\ 2 & \text { if } i=r .\end{cases}
$$

Therefore by assumption of induction hypothesis every edge having one terminal vertex from $V_{i}(1 \leq i \leq k-1)$ lies in the geodesic joining a pair of vertices of $V_{i}(1 \leq i \leq k-1)$. Since the length of the geodesic $v_{i j}-v_{r s}$ 
through the edge $v_{k i} v_{(k+1) j}\left(1 \leq i \leq r_{k}, 1 \leq j \leq r_{k-1}\right)$ is 3 , the edges $v_{k i} v_{(k+1) j}\left(1 \leq i \leq r_{k}, 1 \leq j \leq r_{k-1}\right)$ doesn't lie on a geodesic of $S_{1}=\cup_{i=1}^{i=k-1} V_{i}$. Then either $S=S_{1} \cup V_{k}$ or $S=S_{1} \cup V_{k+1}$ is an edge geodetic set of $G$. Since $r_{k} \leq r_{k+1}, S=S_{1} \cup V_{k}$ is the minimum edge geodetic set of $G$ so that $g_{e}(G)=\sum_{i=1}^{k} r_{i}$. Hence by mathematical induction $g_{e}(G)=\sum_{i=1}^{n-1} r_{i}$.

Theorem 2.27. Let $G=K_{r_{1}, r_{2}, r_{3}, \ldots, r_{n}}$ be a complete n-partite graph. Then $\pi_{s g_{e}}(G)=\max \left\{r_{1}, r_{2}, r_{3} \ldots, r_{n}\right\}$.

Proof. Let us prove this theorem by induction on $n$. Let $n=2$. Then $G$ is a complete bipartite graph. By Theorem 2.25, the result is true for $n=2$. Assume that the result is true for $n=k$. Let $G^{\prime}$ be a complete $k$-partite graph $K_{r_{1}, r_{2}, r_{3}, \ldots, r_{n}}$ and $\pi_{s g_{e}}(G)=\max \left\{r_{1}, r_{2}, \ldots, r_{k}\right\}$. Let $V_{1}=\left\{v_{11}, v_{12}, \ldots, v_{1 r_{1}}\right\}$, $V_{2}=\left\{v_{21}, v_{22}, \ldots, v_{2 r_{2}}\right\}, \ldots, V_{k}=\left\{v_{k 1}, v_{k 2} \ldots, v_{k r_{k}}\right\}$ be the partition of $V$ of a complete $k$-partite graph $G^{\prime}$ such that $r_{1} \leq r_{2} \leq r_{3} \leq \ldots \leq r_{k}$. Let $V_{k+1}=\left\{v_{(k+1) 1}, v_{(k+1) 2} \ldots, v_{(k+1) r_{k+1}}\right\}$. Then $G=G^{\prime}+V_{k+1}$ is a complete $k+1$-partite graph. By Lemma 2.26,

$$
g_{e}(G)=\sum_{i=1}^{k} r_{i} .
$$

Since the $\operatorname{deg}\left(v_{i}\right)=\sum_{i=1}^{k} r_{i}$ for all $v_{i} \in V_{k+1}$ and $V_{k+1}$ is an independent set, by Theorem 2.9, $\pi_{s g_{e}}(G) \geq$ $k+1$. Let $\pi=\left\{H_{1}, H_{2}, \ldots, H_{r_{k}}, H_{r_{k+1}}\right\}$, where $\left\{H_{i}(1 \leq i \leq k)\right\}$ be the star subgraphs having rooted vertex $v_{(k+1) r_{i}}(1 \leq i \leq k)$ and $H_{r_{k+1}}=\left\langle V-\left\{v_{(k+1) 1}, v_{(k+1) 2}, \ldots, v_{(k+1) r_{k}}\right\}\right\rangle$. Since each $H_{i}(1 \leq i \leq k+1)$ is a subgraph having exactly one universal vertex of degree

$$
\sum_{i=1}^{k} r_{i}
$$

for $(1 \leq j \leq k+1)$,

$$
g_{e}\left(H_{j}\right)=\sum_{i=1}^{k} r_{i}
$$

and hence $\pi$ is an edge geodetic decomposition of $G$. Moreover by Lemma 2.26, $\left\langle V-V_{k+1}\right\rangle$ is a connected subgraph with edge geodetic number

$$
g_{e}(G)=\sum_{i=1}^{(k-1)} r_{i} .
$$

Hence, $\pi$ be a maximum edge geodetic self decomposition so that $\pi_{s g_{e}}(G)=r_{k+1}=\max \left\{r_{1}, r_{2}, r_{3}, \ldots, r_{k+1}\right\}$. Hence, the result is true for $n=k+1$. Therefore by induction hypothesis the result is true for all $n$.

Corollary 2.28. For the complete r-partite graph $G=K_{r_{1}, r_{2}, r_{3}, \ldots, r_{n}}\left(r_{i}>2\right), \pi_{s g_{e}}(G)+g_{e}(G)=p$.

Proof. This follows from Lemma 2.26 and Theorem 2.27 .

Theorem 2.29. For any connected graph $G, \pi_{s g_{e}}(G) g_{e}(G)=q$ and $\pi_{s g_{e}}(G)+g_{e}(G)=p$, if and only if $G=K_{m, n},(3 \leq m \leq n)$.

Proof. Suppose that $\pi_{s g_{e}}(G) g_{e}(G)=q$ and $\pi_{s g_{e}}(G)+g_{e}(G)=p$. By Theorem 2.14, it is enough to prove that $G_{i}=K_{1, m}$, if and only if $G=K_{m, n}(m \leq n)$. Let $V=\left\{v_{1}, v_{2} \ldots, v_{m}\right\}$ and $W=\left\{w_{1}, w_{2} \ldots, w_{n=p-m}\right\}(m \leq n)$. Without loss of generality assume that $v_{i} \neq w_{j}(1 \leq i \leq m, 1 \leq j \leq n)$. To prove $U$ and $V$ are the partition of $V(G)$. Suppose this not the case. Then $G$ has at least two adjacent vertices $v_{i}$ and $v_{j}$ from $V$ or some adjacent vertices from $W$. Moreover $v_{i}, v_{j}$ and $w_{l}(1 \leq l \leq n)$ form a triangle so that $w_{l}$ is an extreme vertex of $G$. Then $w_{l}$ belongs to every minimum edge geodetic set of $G$. Hence, $g_{e}(G)>m \neq g_{e}\left(G_{i}\right)$ and $\pi_{s g_{e}}(G)<p-g_{e}(G)$, which is a contradiction. The converse follows from Theorems 1.3, 2.14 and 2.25. 
Theorem 2.30. Let $G$ be a bipartite graph. Then $\pi_{s g_{e}}(G)=g_{e}(G)$, if and only if $G=K_{m, m}(m \geq 3)$ or $K_{1,2}$.

Proof. Let $G$ be a bipartite graph and $\pi_{s g_{e}}(G)=g_{e}(G)$. Suppose that $G=K_{m, n}(n>m)$ and $G \neq K_{1,2}$. Then by Theorems 1.3 and $2.25, \pi_{s g_{e}}(G) \neq g_{e}(G)$, which is a contradiction. Hence, $G=K_{m, m}(m \geq 2)$ or $K_{1,2}$. Conversely suppose that $G=K_{m, m}(m \geq 2)$. Theorems 1.3 and $2.25, \pi_{s g_{e}}(G)=g_{e}(G)$. Also, if $G=K_{1,2}$ then $\pi=\left\{K_{2}, K_{2}\right\}$ and by Theorem 1.2, $\pi$ is the unique edge geodetic self decomposition of $G$. Thus $\pi_{s g_{e}}(G)=g_{e}(G)$.

We have the following realisation theorem.

Theorem 2.31. For any positive integer $3 \leq a \leq b$, there exists a connected graph $G$ such that $g_{e}(G)=$ $a, \pi_{s g_{e}}(G)=b$.

Proof. Case i. Suppose that $3 \leq a<b$. Let $G=K_{a, b}(3 \leq a<b)$. Then by Theorems 1.3 and $2.25, g_{e}=a$, $\pi_{s g_{e}}(G)=b$.

Case ii. Suppose that $2<a=b$. Let $G=K_{a, a}(a \geq 3)$. Then by Theorem $2.30, g_{e}(G)=a, \pi_{s g_{e}}(G)=a$.

Acknowledgements. The authors are grateful to the referees whose valuable comments resulted in an improved paper.

\section{REFERENCES}

[1] P. Arul Paul Sudhahar, A. Ajitha and A. Subramanian, Edge geodetic domination number of a graph. Int. J. Math. App. 4 (2016) 45-50.

[2] M. Atici, On the edge geodetic number of a graph. Int. J. Comput. Math. 80 (2003) 853-861.

[3] F. Buckley and F. Harary, Distance in Graphs. Addison-Wesley, Redwood City, CA (1990).

[4] F. Harary, Graph Theory. Narosa Publishing House, New Dehli (1998).

[5] J. John and D. Stalin, Edge geodetic self decomposition of graphs. Disc. Math. Algorithms App. 12 (2020) 2050064.

[6] R.E. Mariano and S.R. Canoy, Jr., Edge geodetic covers in graphs. Int. Math. Forum 46 (2009) $2301-2310$.

[7] P. Paulraja and S. Ganesamoorthy, Multidecompositions of line graphs of complete graphs. Disc. Math. Algorithms App. 11 (2019) 1950035.

[8] P. Paulraja and T. Sivakaran, Decompositions of some regular graphs into unicyclic graphs of order five. Disc. Math. Algorithms App. 11 (2019) 1950042.

[9] V. Samodivkin, On the edge geodetic and edge geodetic domination numbers of a graph. Commun. Comb. Optim. 5 (2019) $41-54$.

[10] A.P. Santhakumaran and J. John, Edge geodetic number of a graph. J. Disc. Math. Sci. Cryptography 10 (2007) 415-432.

[11] A.P. Santhakumaran and J. John, The connected edge geodetic number of a graph. Scientia 17 (2009) 67-82.

[12] A.P. Santhakumaran and J. John, The upper edge geodetic number and the forcing edge geodetic number of a graph. Opuscula Math. 29 (2009) 427-441.

[13] A.P. Santhakumaran and J. John, The upper connected edge geodetic number of a graph. Filomat 26 (2012) $131-141$.

[14] A.P. Santhakumaran and S.V. Ullas Chandran, Comment on "Edge geodetic covers in graphs". Proyecciones J. Math. 34 (2015) 343-350.

[15] D. Stalin and J. John, Edge geodetic dominations in graphs. Int. J. Pure Appl. Math. 116 (2017) 31-40.

[16] D. Stalin and J. John, Forcing edge geodetic dominations in graphs. Int. J. Pure Appl. Math. 10 (2018) $172-177$.

[17] D. Stalin and J. John, Upper edge geodetic dominations in graphs. J. Adv. Res. Dyn. Control Syst. 9 (2018) $201-205$.

[18] F. Zheng, Advanced hybrid approaches based on graph theory decomposition, modified evolutionary algorithm and deterministic optimisation techniques for the design of water distribution systems, Ph.D. thesis. The University of Adelaide (2013).

[19] F. Zheng, A.C. Zecchin and A.R. Simpson, A decomposition and multistage optimization approach applied to the optimization of water distribution systems with multiple supply sources. Water Resour. Res. 49 (2012) 380-399. 